\title{
Spontaneous closure of a large atrial septal defect in an infant
}

\author{
Roland Fiszer ${ }^{1}$, Małgorzata Szkutnik², Beata Chodór ${ }^{1}$, Jacek Białkowski² \\ ${ }^{1}$ Silesian Center for Heart Diseases, Zabrze, Poland \\ ${ }^{2} \mathrm{~S} c h$ ool of Medicine with Division of Dentistry, Medical University of Silesia, Zabrze, Poland
}

Postep Kardiol Inter 2014; 10, 4 (38): 264-266

DOI: $10.5114 /$ pwki.2014.46768

\begin{abstract}
A bstract
We describe a patient who, at the age of 6 months (body weight $6.1 \mathrm{~kg}$ ), was diagnosed with a large atrial septal defect (with diameter 10-11 mm) that had spontaneously closed in a 1-year period. The timing of transcatheter intervention in children should be considered individually, paying close attention to subsequent echocardiographic data.
\end{abstract}

Key words: atrial defect, spontaneous closure.

\section{Introduction}

Atrial septal defect occurs at an incidence rate 1 per 1500 live births, and nowadays the preferred method of treatment is transcatheter closure. Many previous reports regarding natural history of small atrial defects have described spontaneous closure [1-6]. However, large defects usually increase in size with age [7, 8]. We describe young patient in whom spontaneous closure of a large atrial septal defect occurred.

\section{Case report}

In April of 2013, a 6-month-old girl was referred for clinical evaluation to our centre. The infant was born at term weighing $2.5 \mathrm{~kg}$ and had Apgar score 9 and 10 at 1 and 5 min, respectively. The atrial septal defect was diagnosed in another hospital where the infant was treated because of infection of the urinary tract at the age of 1.5 months of life. The parents denied any history of poor feeding, cyanosis, increased respiratory tract infections, or failure to thrive. They noted only increased diaphoresis of the child.

Physical examination demonstrated an active, noncyanotic infant in no distress with body weight $6.1 \mathrm{~kg}$. The heart rate was $130 / \mathrm{min}$, respiratory rate $48 / \mathrm{min}$, systemic oxygen saturation $98 \%$ in room air, and blood pressure in the right arm was $78 / 43 \mathrm{~mm} \mathrm{Hg}$. Cardiac examination revealed hyperactive precordium with normal first and split second heard sound and a grade II-III/VI systolic ejection murmur at the left upper sternal border. The liver was $2 \mathrm{~cm}$ below the right costal margin. The pulses were of normal volume and equal in the upper and lower extremities. Chest radiography demonstrated levocardia, a cardiothoracic ratio of 0.5 , and increased pulmonary vascularity. An electrocardiogram demonstrated sinus rhythm with partial block of the right bundle His branch (in V1 rsR' morphology). In transthoracic echocardiography (2D, colour Doppler), a centrally located defect was demonstrated with diameter 10-11 mm (index diameter of defect/weight $=1.42$ ) with left-to-right shunting with paradoxical movement of the interventricular septum, enlargement of the right atrium and ventricle (right ventricle diastolic diameter $-1.64 \mathrm{~cm}$ ), and relatively small left ventricle (diameters of left ventricle-diastole/diameters of left ventricle - systole, respectively, 2.04/1.18 cm). Pulmonary flow was augmented. Captropril, spironol, and furosemide were introduced as pharmacotherapy. When the child was re-evaluated in the age of 1 year, her weight was $7.9 \mathrm{~kg}$ (15-50 percentile). She was asymptomatic. An echocardiogram demonstrated a $9 \mathrm{~mm}$ defect (index diameter of defect/weight $=1.12$ ) with augmented pulmonary flow. Gradient from small tricuspid regurgitation was $27 \mathrm{~mm} \mathrm{Hg}$. The right heart was enlarged (right ventricle diastolic diameter $-1.57 \mathrm{~cm}$ ), diameters of the left ventricle in diastole/left ventricle in systole, respectively, were $2.1 / 1.3 \mathrm{~cm}$ (Figure 1).

\section{Corresponding author:}

Roland Fiszer MD, Silesian Center for Heart Diseases, 9 Curie-Skłodowskiej St, 41-800 Zabrze, Poland, phone: +48 504125057 ,

e-mail: rfiszer@gumed.edu.pl

Received: 11.07.2014, accepted: 29.09.2014. 


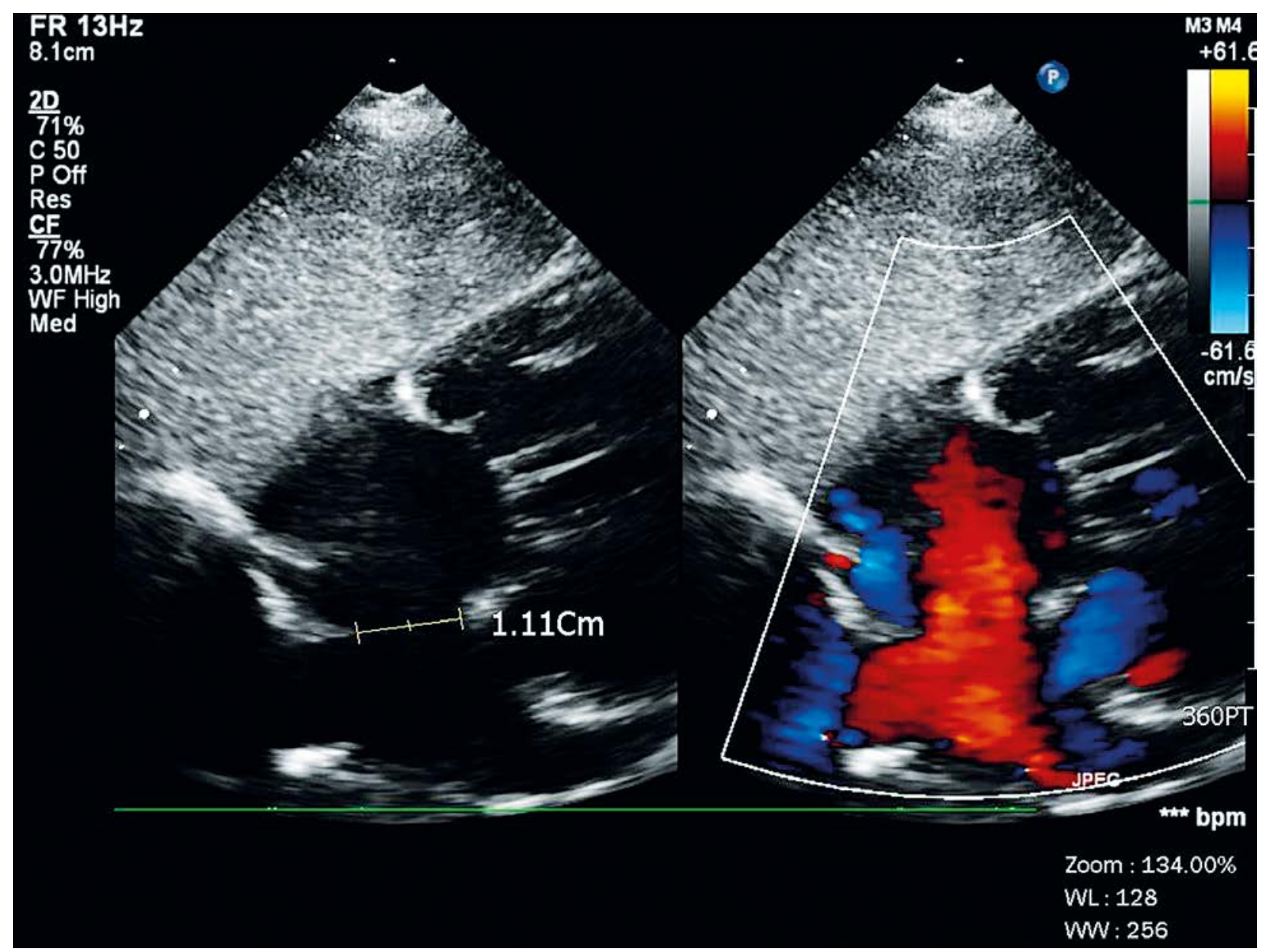

Figure 1. Large atrial septal defect at the age of 6 months

Pharmacotherapy was continued. She was admitted again at the age of 1.5 years with body weight $10 \mathrm{~kg}$. She was asymptomatic and her chest radiography and electrocardiogram was normal. An echocardiogram demonstrated only a trivial shunt through the atrial septum, normal movement of the interventricular septum, and the diameters of the right and left cavities were normal (right ventricle diastolic diameter $=1.00 \mathrm{~cm}$; left ventricle diastolic diameter/left ventricle systolic diameter $=$ 2.67/1.74 cm, respectively) (Figure 2).

Pharmacotherapy was discontinued.

\section{Discussion}

The natural history of secundum atrial septal defect remains neither completely understood nor completely predictable. Spontaneous closure has been described in $100 \%$ of patients with a defect less than $3 \mathrm{~mm}$ at 18 months of age [1]. On the other hand, reports from some facilities [7-9] indicate that the size may become larger while waiting for interventional treatment, and it may deviate from the indications for treatment. Tortoriello et al. [9] reported that a small atrial septal defect (size 3-4 mm) enlarged to $24 \mathrm{~mm}$ after 6 years. Also, Saito et al. [6] treated an infant with a defect size that enlarged from
$9 \mathrm{~mm}$ to $27 \mathrm{~mm}$ over a period of 9 years. In both cases interventional treatment became unsuitable and finished as surgical closure. The rate of defect enlargement ranged from $29 \%$ [8] to $65 \%$ [7]. Saito had no spontaneous closure of atrial defects in his medium sized series in paediatric patients. Generally it is believed that large defects usually enlarge with age [7-9] while small ones close spontaneously [1-6]. Whether atrial septal defect growth is related to the pubertal growth spurt, persistent continuous shunting, or stretching in the opposite direction of the short axis of an elliptoid defect remains unclear [2]. It is also generally believed that increases in diameter are related to increased compliance of the right ventricle, increased blood flow through the defect, and increased right atrium size with age. In our case we observed in subsequent measurements in transthoracic echocardiography a decrease of the right ventricle diameter with concomitant increase of the diameter of the left ventricle. Whether or not this was related to a decrease in right ventricle compliance seems impossible to answer. According to the experience we have obtained from this case, it seems obligatory to assess subsequent echocardiography results before decision-making regarding transcatheter closure of the defect. It is a very 


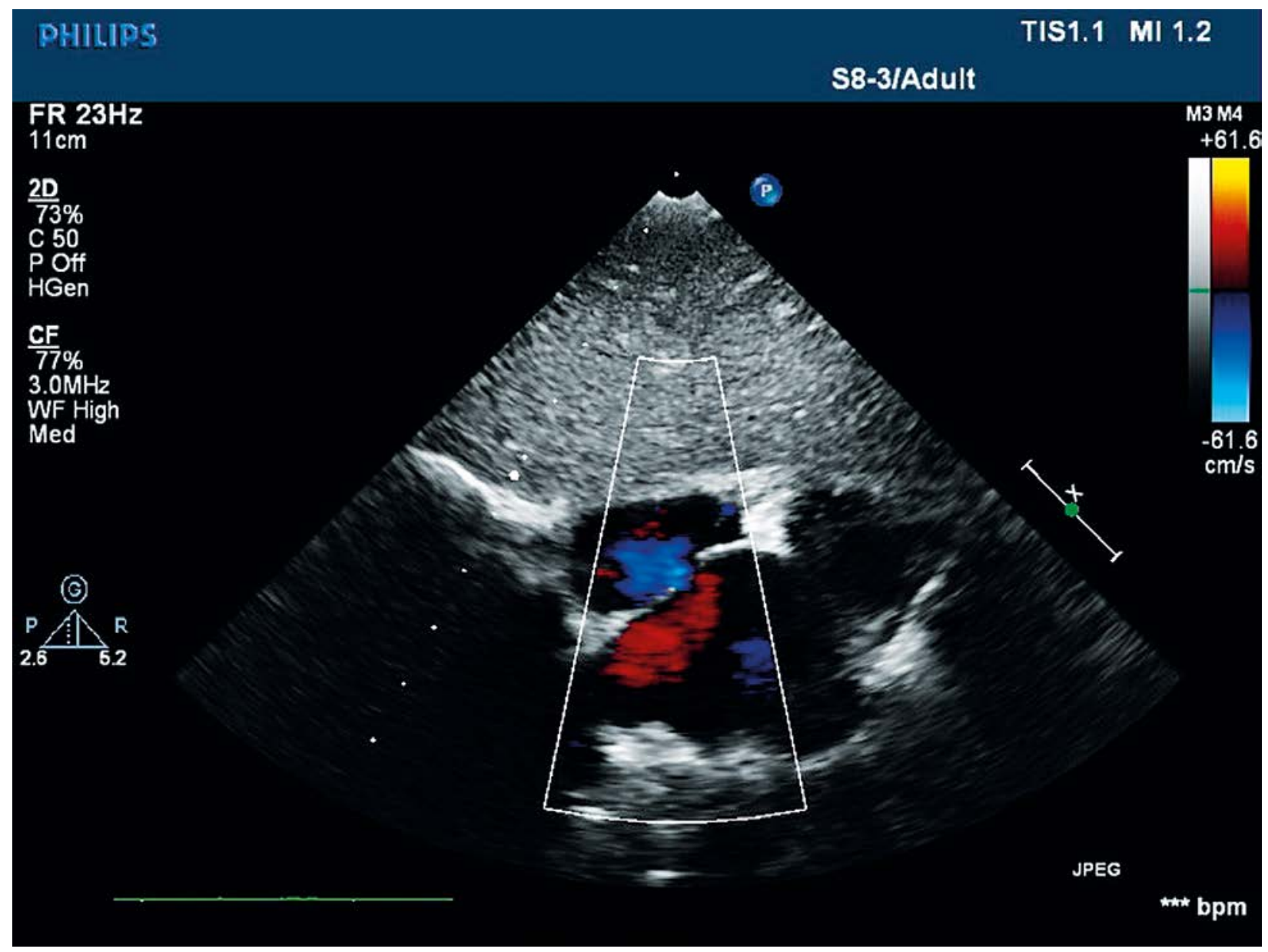

Figure 2. Trivial atrial septal defect at the age of 18 months

important issue because in many centres (also in our department) the tendency to close secundum atrial septal defects in younger children is increasing. Nevertheless, in all of our patients below $10 \mathrm{~kg}$ of body weight, who had closed percutaneously atrial defect, the diameters of the defect in consecutive echo examinations increased and all patients were symptomatic [10].

\section{Conclusions}

Although spontaneous closure of a large secundum atrial septal defect is relatively uncommon, the potential for such a possibility exists. This has significance not only for frequency of follow-up, but also for the timing of a possible transcatheter occlusion of the defect.

\section{References}

1. Radzik D, Davigon A, Van Doesburg N, et al. Predictive factors for spontaneous closure of atrial septal defects diagnosed in the first 3 months of life. J Am Coll Cardiol 1993; 22: 851-3.

2. Helgason H, Jonsdottir G. Spontaneous closure of atrial septal defects. Pediatr Cardiol 1999; 20: 195-9.

3. Azhari N, Shihata MS, Al-Fatani A. Spontaneous closure of atrial septal defects within the fossa ovale. Cardiol Young 2004; 14: 148-55.
4. Hanslik A, Pospisll U, Salzer-Muhar U, et al. Predictors of spontaneous closure of isolated secundum atrial septal defect in children: a longitudinal study. Pediatrics 2006; 118: 1560-5.

5. Demir T, Ostund T, Eroglu AG, et al. Outcome for patients with isolated atrial septal defect in the oval fossa diagnosed in infancy. Cardiol Young 2008; 18: 75-8.

6. Saito T, Ohata K, Nakayama Y, et al. Natural history of medium-sized atrial septal defects in pediatric cases. J Cardiol 2012; 60: 248-51.

7. McMahon CJ, Feltes TF, Fraley JK, et al. Natural history of growth of secundum atrial septal defect and implications for transcatheter closure. Heart 2002; 87: 256-9.

8. Saxena A, Divekar A, Soni NR. Natural history of secundum atrial septal defects revisited in the era of transcatheter closure. Indian Heart J 2005; 57: 35-8.

9. Tortoriello TA, McMahon C, Kovalchin JP, et al. Growth of an atrial septal defect: missing the window for transcather closure. Pediatr Cardiol 2002; 23: 542-4.

10. Knop M, Szkutnik M, Fiszer R, et al. Transcatheter closure of atrial septal defect in children up to $10 \mathrm{~kg}$ of body weight with Amplatzer device. Cardiology J 2014; 21: 279-83. 